\title{
Canal wall reconstruction - surgical considerations
}

\author{
D.C. GHEORGHE ${ }^{1,2}$, A. ZAMFIR-CHIRU-ANTON ${ }^{3}$ \\ ${ }^{1}$ ENT Department, "M.S. Curie” Children Hospital, Bucharest \\ 2"Carol Davila" University of Medicine and Pharmacy, Bucharest \\ ${ }^{3}$ ENT Department, "Grigore Alexandrescu" Children Hospital, Bucharest
}

\begin{abstract}
Recurrent cholesteatoma and local mastoid morbidity account for common patient complaints in our pediatric department.

Objective. To describe a surgical technique used for 2 difficult cholesteatoma pediatric cases, in order to prevent mastoid cavity associated disease.

Material. 2 cases with recurrent cholesteatoma and mastoid morbidity were surgically approached by reconstructing the posterior osseous canal wall.

Results. Anatomical results were satisfactory on short term monitoring. Long-term results need further evaluation.

Conclusion. Posterior canal wall reconstruction represents a useful option for mastoid cavity patients with local recurrent disease.
\end{abstract}

Keywords: radical mastoidectomy, recurrent cholesteatoma, posterior canal wall reconstruction

Infectious diseases of the middle ear and mastoid can sometimes lead to bone erosion and chronic mucosal infection. These cases usually need a surgical approach. The common techniques involve either preservation of the external ear canal (canal wall up) (1) of removing it (canal wall down). (2) The advantages and difficulties that arise from both methods have been described by many papers. (3)

Open technique has been associated with low disease reccurence but less optimal functional results. (4) Lack of self cleaning and restriction of water contact with it, makes the open technique a less desirable option. A lot of reasons can be responsible for its performing: advanced inflammatory disease of the mastoid bone, low medical addressability, low patient monitoring. (5) It must also be noted that during the last years, more papers communicated similar functional results to those obtained by closed technique. (6)

Due to recent surgeries of 2 cholesteatoma operated cases, in which recurrent disease was discovered, we started reconstructions of the mastoid cavity, using autologous cortical temporal bone. We present here the technique and the first results. 


\section{CASE PRESENTATIONS}

\section{Case 1}

A patient aged 9, operated 2 years before is admitted to our department for investigations. He has been diagnosed previously with cholesteatoma of the mastoid and middle ear. The disease had been removed by radical mastoidectomy with tympanic membrane reconstruction with autologous cartilage and pericondrium. A total ossicular replacement prothesis had been installed 1 year after the cholesteatoma removal, followed by $20 \mathrm{~dB}$ hearing gain.

After 1 more year the patient noticed a deterioration of its hearing thereshoulds in his operated ear. The decision to reoperate was taken. Intraoperatively, we discovered cholesteatoma recurrence, originating from the old mastoid cavity, with displacement of the ossicular prothesis from its place by the epidermal growth. Total removal of recurrent cholesteatoma was followed by posterior ear canal reconstruction, using a graft harvested from the cortical temporal squamous bone, just behing the mastoid cavity edge. It was fixed into position with glass ionomer cement (Ketac Cem ${ }^{R} 3 \mathrm{M}$ ESPE). Tympanic membrane was reconstructed using composite graft and a new TORP was installed again. A Palva flap covered the anterior face of the reconstructed bony ear canal. Recovery from surgery went uneventful and the patient is in close monitoring for its cholesteatomatous disease.

\section{Case 2}

A 16 year old child had been previously operated for extensive middle ear and mastoid cholesteatoma 3 years before (radical mastoidectomy). The follow-up of the patient revealed a large cavity, without self-cleansing capabilities.
As a consequence the patient was consulted at a 6 months basis, for close monitoring. Although he was indicated for a new surgical exploration, he refused until the last examination revealed a poorly controled cavity, with some minor sustained discharge. We performed a new surgery wich revealed a recurent cholesteatoma of the cavity. After thorough removal of the disease we proceeded to the reconstruction of the osseous external ear canal, with cortical bone harvested from the edge of the mastoid cavity. It was fixed with glass ionomer cement. Tympanic membrane reconstration and Palva flap were part of the surgery in the end. Recovery went without any complications. The patient remained to be monitored for recurrent disease in the future.

\section{DISCUSSION}

Total eradication of middle ear cholesteatoma can be challenging in the absence of a good view upon the facial recess and sinus tympani. Removal of the bony posterior canal wal can ensure a good approach in all cases. (7)

Radical mastoidectomy (canal wall down procedures) is a well known source of local morbidity for the patient in long-term surveillance. Due to the lack of self cleansing possibility and sometimes disproportionate dimensions related to the external meatus, recurrent otorrheea and cholesteatoma can occur. The decision to perform radical surgery from the beginning is also debatable. It is our opinion that if conservative management is achievable, we go for it. Still, most of our patients come to the ENT department with advanced disease cholesteatoma, making its removal very difficult in the presence of an intact posterior canal wall. Performing
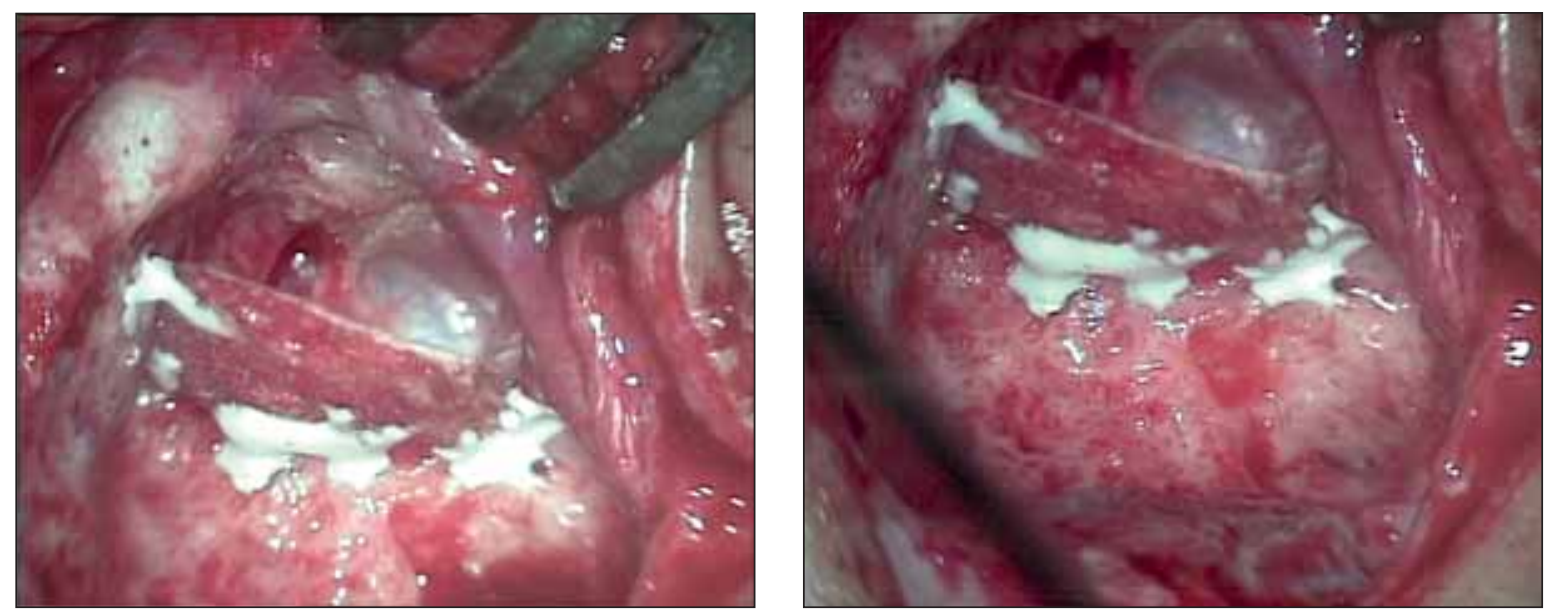

FIGURE 1. Osseous plate fixed inposition with glass ionomer cement 
a very large plasty of the external meatus can sometimes prevent such problems. Local comblating flaps (like Palva) can also be of use. Still, the young age of pediatric patients predisposes to recurrent disease. That is why we decided to reconstruct the posterior canal wall in our patients, in order to obtain a disease free mastoid bone.

The usefullness of radical surgery can be counteracted by different means within surgical techniques development. Temporary removal of the posterior canal wall and endoscopy were equally described to ensure proper removal of all cholesteatoma from the middle ear spaces and prevent recurrence. (8) We could consider their use as well, if the extent of the disease associated osteitis would not afflict the bone of the canal wall.

The reason for advanced disease observed in our department could be accounted because of the lack of periodic hearing screening from the medical health care system. Also, some medical addressability issues could be responsible for some cases admited in our service.

Reconstruction of the canal wall was described and used in the past. (9) It can be used as a single procedure in cholesteatoma treat- ment with acceptable results. (10) Different materials have been proposed to perform this kind of technique. (11)

We think that the reconstruction technique of the posterior canal wall is useful just for some of our radical mastoid surgery patients but not all of them. Local morbidity and lack of full visual control of the cavity are good indications for this kind of surgery. Patients that do not cooperate to close follow-up can also be good candidates. Decision to choose between local comblating flaps and osseous reconstruction depends clearly on the surgeon. It also remains to debate if single stage surgery is to be performed at the initial cholesteatoma removal, taking anatomical/inflammatory conditions into account.

\section{CONCLUSION}

Difficult cholesteatoma cases that require extensive initial surgery and can not be subsequently monitored on a regular basis can benefit osseous reconstruction of the posterior canal wall as part of their recovery and long-term disease prevention. The technique is easily performed and reproducible. Long-term results remain to be communicated in our next papers.

\section{REFERENCES}

1. Darrouzet V., et al. Preference for the closed technique in the management of cholesteatoma of the middle ear in children: a retrospective study of 215 consecutive patients treated over 10 years. Am J Otol, 2000. 21(4): p. 474-81.

2. Sun J., et al. Canal wall-down mastoidectomy with mastoid obliteration for pediatric cholesteatoma. Acta Otolaryngol, 2010. 130(2): p. 259-62.

3. Walker P.C., et al. Long-term results of canal wall reconstruction tympanomastoidectomy. Otol Neurotol, 2014. 35(1): p. e24-30.

4. Yung M., Jacobsen N.L., Vowler S.L. A 5-year observational study of the outcome in pediatric cholesteatoma surgery. Otol Neurotol, 2007. 28(8): p. 1038-40.
5. Nash R., et al. Canal wall reconstruction and preservation in the surgical management of cholesteatoma in children with Down's syndrome. Int J Pediatr Otorhinolaryngol, 2014. 78(10): p. 1747-51.

6. Bacciu A., et al. Surgical treatment of middle ear cholesteatoma in children with Down syndrome. Otol Neurotol, 2005. 26(5): p. 1007-10.

7. Vercruysse J.P., et al. Mastoid and epitympanic bony obliteration in pediatric cholesteatoma. Otol Neurotol, 2008. 29(7): p. 953-60.

8. Ayache S., Tramier B., Strunski V. Otoendoscopy in cholesteatoma surgery of the middle ear: what benefits can be expected? Otol Neurotol, 2008. 29(8): p. 1085-90.
9. Gantz B.J., Wilkinson E.P., Hansen M.R., Canal wall reconstruction tympanomastoidectomy with mastoid obliteration. Laryngoscope, 2005. 115(10): p. 1734-40.

10. Dornhoffer J.L. Retrograde mastoidectomy with canal wall reconstruction: a follow-up report. Otol Neurotol, 2004. 25(5): p. 653-60.

11. Zini C., Quaranta N., Piazza F. Posterior canal wall reconstruction with titanium micro-mesh and bone pate. Laryngoscope, 2002. 112(4): p. 753-6. 